\title{
Unusual Stacking Variations in Liquid-Phase Exfoliated Transition Metal Dichalcogenides
}

\author{
Aleksey Shmeliov, ${ }^{\dagger}$ Mervyn Shannon, ${ }^{\ddagger}$ Peng Wang, ${ }^{\S}$ Judy S. Kim, ${ }^{\dagger}$ Eiji Okunishi, ${ }^{\perp}$ Peter D. Nellist, ${ }^{\dagger}$ \\ Kapildeb Dolui," Stefano Sanvito," and Valeria Nicolosi" ${ }^{\uparrow, *}$
}

\begin{abstract}
${ }^{\dagger}$ Department of Materials, University of Oxford, Parks Road, 0X1 3PH 0xford, United Kingdom, ${ }^{\ddagger}$ STFC Daresbury Laboratories, SuperSTEM, Keckwick Lane, WA4 4AD Warrington, United Kingdom, ${ }^{5}$ National Laboratory of Solid State Microstructures and Department of Materials Science and Engineering, Nanjing University, Nanjing 210093, People's Republic of China, ${ }^{\perp}$ EM Application Group, EM Business Unit, JEOL Ltd., 1-2 Musasino 3-chome Akishima, Tokyo 196-8558, Japan, and

"Schools of Physics and "Schools of Chemistry and Physics, CRANN, AMBER, Trinity College Dublin, Dublin 2, Ireland
\end{abstract}

\begin{abstract}
Liquid-phase exfoliation of layered materials offers a large-scale approach toward the synthesis of $2 D$ nanostructures. Structural properties of materials can however change during transition from bulk to the 2D state. Any such changes must be examined and understood for successful implementation of $2 \mathrm{D}$ nanostructures. In this work, we demonstrate nonbulk stacking sequences in the few-layer $\mathrm{MoS}_{2}$ and $\mathrm{WS}_{2}$ nanoflakes produced by liquid-phase exfoliation. Our analysis shows that nonbulk stacking sequences can be derived from its bulk counterparts by translational shifts of the layers. No structural changes within the layers were observed. Twenty-seven $M_{0} S_{2}$ and five WS $S_{2}$ nanoflakes were imaged and analyzed. Nine $\mathrm{MoS}_{2}$ and four $W_{2}$ nanoflakes displayed nonbulk stacking. Such dominance of the nonbulk stacking suggests high possibility of unusual stacking sequences in other $2 \mathrm{D}$ nanostructures. Notably, the electronic structure of some non bulk

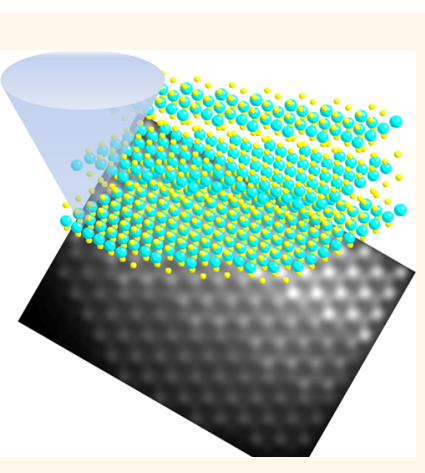
stacked bilayers presents characteristics which are uncommon to either the bulk phase or the single monolayer, for instance, a spin-split conduction band bottom. Our main characterization technique was annular dark-field scanning transmission electron microscopy, which offers direct and reliable imaging of atomic columns. The stacking characterization approach employed here can be readily applied toward other few-layer transition metal chalcogenides and oxides.
\end{abstract}

KEYWORDS: transition metal dichalcogenides $\cdot \mathrm{MoS}_{2} \cdot \mathrm{WS}_{2} \cdot$ two-dimensional nanomaterials . aberration-corrected scanning transmission electron microscopy

\begin{abstract}
$\Lambda$ tomically thin layered crystals have emerged as a new class of two-dimensional (2D) nanomaterials with high specific surface area, important for a wide range of applications ${ }^{1,2}$ ranging from electronics ${ }^{3-5}$ to sensing, ${ }^{6,7}$ catalysis, ${ }^{8}$ energy harvesting, ${ }^{9,10}$ and storage. ${ }^{11,12}$ While graphene is the most renowned $2 \mathrm{D}$ material, there has been considerable recent interest in the exfoliation of other layered compounds such as transition metal dichalcogenides (TMDs). TMDs consist of hexagonal layers of metal atoms (M) sandwiched between two layers of chalcogen atoms $(X)$ with $\mathrm{MX}_{2}$ stoichiometry. Although the planar bonding within each of these three atomic layers is covalent, sheets stack via van der Waals interactions to form a 3D crystal. TMDs occur in more than 40 different types, depending on the combination of chalcogen ( $\mathrm{S}, \mathrm{Se}$, or $\mathrm{Te}$ ) and transition
\end{abstract}

metal. ${ }^{13}$ Depending on the coordination and oxidation state of the metal atoms, TMDs can be metallic, semimetallic, or semiconducting. ${ }^{14,15}$ Like graphene, 2D TMDs have attracted considerable attention due to the wide range of unique properties unlocked by exfoliation such as enhanced surface area, reactivity, and quantum confinement of electrons, after neighboring layers are removed, which creates in essence unique physical and chemical properties. $\mathrm{MoS}_{2}$ and $\mathrm{WS}_{2}$ are for example, semiconductors with a band gap in the range of $1.1-1.7 \mathrm{eV}^{16}$ and were among the first layered materials, which were attempted to be exfoliated down to single layers. ${ }^{17-19} \mathrm{MoS}_{2}$ and $\mathrm{WS}_{2}$ have an indirect band gap in its 3D bulk form and become direct gap, fluorescent 2D semiconductors in the limit of complete exfoliation. $^{20-22}$
*Address correspondence to
nicolov@tcd.ie.

Received for review January 18, 2014 and accepted March 3, 2014.

Published online March 03, 2014 $10.1021 /$ nn5003387

(C) 2014 American Chemical Society 


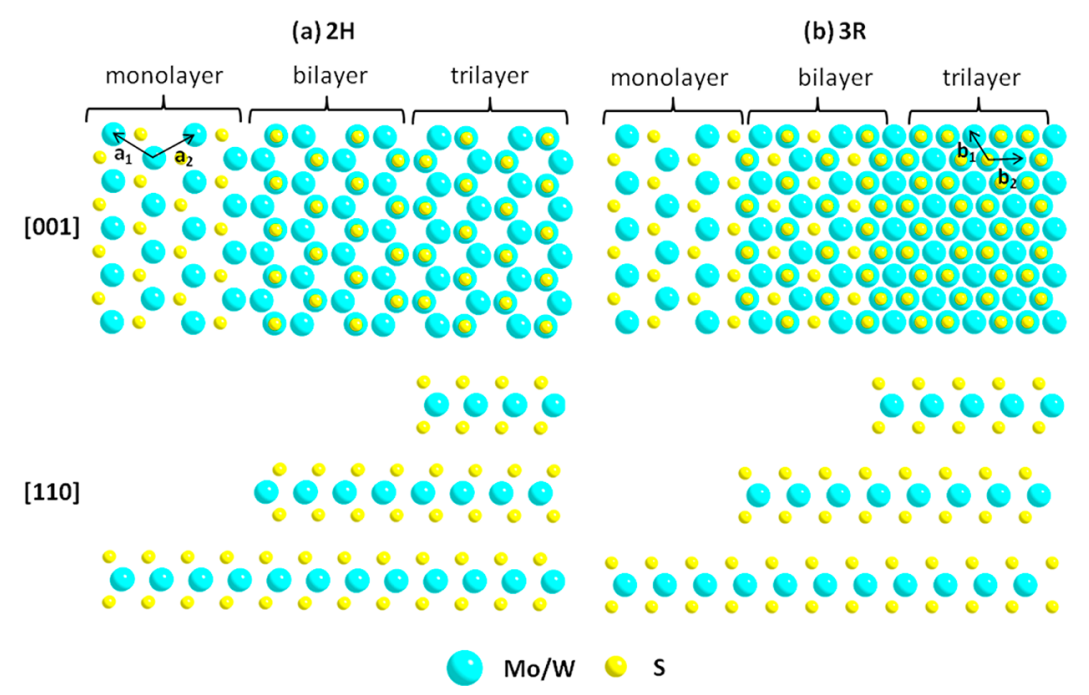

Figure 1. Schematic of the $\mathrm{MoS}_{2}$ and WS $\mathrm{S}_{2}$ (a) $2 \mathrm{H}$ and (b) $3 \mathrm{R}$ polytypes. Views along [001] direction show the projected features expected to be observed in the experimental ADF STEM images. Views along [110] show unambiguously the alternative stacking sequences correlated with the two different polytypes. Vectors $a_{1}, a_{2}$ and $b_{1}, b_{2}$ represent position vectors of the 2D hexagonal Bravais lattice formed by the atomic columns; $\left|a_{1}\right|=\left|a_{2}\right|=3.16 \AA$ and $\left|b_{1}\right|=\left|b_{2}\right|=1.83 \AA$ for $\operatorname{MoS}_{2} ;\left|a_{1}\right|=\left|a_{2}\right|=3.15 \AA$ and $\left|b_{1}\right|=\left|b_{2}\right|=1.82 \AA$ for $W S_{2}$.

The main production method of TMD nanosheets until a few years ago involved intercalation with inorganic or organic species. ${ }^{19,23}$ Intercalation reactions are however mostly based on laborious and time-consuming chemical processes and are only semiscalable. Recent advances in liquid-phase exfoliation opened new opportunities for large-scale applications. ${ }^{24}$ With this method, high-concentration dispersions (up to $0.3 \mathrm{~g} \mathrm{~L}^{-1}$ ) of $\mathrm{MoS}_{2}, \mathrm{WS}_{2}, \mathrm{TiS}_{2}, \mathrm{MoSe}_{2}, \mathrm{MoTe}_{2}, \mathrm{TaSe}_{2}$, $\mathrm{NbSe}_{2}, \mathrm{NiTe}_{2}$, and $\mathrm{Bi}_{2} \mathrm{Te}_{3}$ were obtained in various organic solvents in complete absence of intercalants ${ }^{24}$ as well as in water with the aid of common surfactants. ${ }^{25}$ Two-dimensional TMD materials prepared by this scalable method have already attracted considerable interest for applications in composites ${ }^{24}$ and energy storage. ${ }^{26}$ Another promising application of exfoliated TMDs is in nanoelectronics. ${ }^{3}$ Single-layer $\mathrm{MoS}_{2}$ has been proven to fabricate transistors with high mobility ( $>200 \mathrm{~cm}^{2} / \mathrm{Vs}$ and sometimes as high as $780 \mathrm{~cm}^{2} / \mathrm{Ns}$ ) and high current on/off ratios $\left(>10^{8}\right) .^{3}$ While these transistors were based on scotch-tape exfoliated $\mathrm{MoS}_{2}$, scalable liquid-phase exfoliation methods are envisaged to be required for larger-scale applications. In this context, studying the atomic structure of the exfoliated compounds becomes crucial for understanding the materials' behavior and predicting their performance when used in composites, catalysts, nanoelectronics, and energy storage/harvesting devices.

Most of the initial techniques employed for the characterization of intercalated and chemical vapor deposition (CVD)-grown nanosheets were X-ray diffraction, ${ }^{19,23,27}$ Raman, ${ }^{27,28}$ absorption fine spectroscopies, ${ }^{29,30}$ and scanning tunneling microscopy (STM). ${ }^{31,32}$ Only recently, due to advances in aberration-corrected microscopy, the first structural study based on atomically resolved annular dark-field (ADF) scanning transmission electron microscopy (STEM) images appeared in the literature. ${ }^{33-38}$ The use of ADF STEM is particularly effective in the study of $2 \mathrm{D}$ nanosheets as the incoherent nature of the imaging process provides directly interpretable atomic resolution, and the relatively high angles of scattering provide image intensities strongly dependent on atomic number, $Z{ }^{39}$ This enables direct observation and identification of the sublattices, as well as substitutional defects and stacking faults. In this work, we present a comprehensive structural characterization of 2D TMD nanosheets produced by liquid-phase exfoliation by a combination of high spatial resolution aberration-corrected ADF STEM and image simulation. In particular, we focus on $\mathrm{MoS}_{2}$ and $\mathrm{WS}_{2}$, which are two of the most commercially relevant TMD compounds. This work differs from previous studies of this type exclusively focused on nanosheets produced by nonscalable and less industrially exploitable methods such as intercalation 19,23,27,29-32 and/or micromechanical cleavage $\mathrm{e}^{17,28,40}$ and complements characterization of the samples produced by chemical vapor deposition. $^{35,41}$

$\mathrm{MoS}_{2}$ and $\mathrm{WS}_{2}$ are isostructural with two naturally occurring polytypes $2 \mathrm{H}$ and $3 \mathrm{R}$ (Figure 1). The coordination of the metal ion is trigonal prismatic (Figure 1). A nonbulk 1T polytype was also reported for 2D $\mathrm{MoS}_{2}$ and $\mathrm{WS}_{2}$ nanocrystals prepared via intercalation. ${ }^{42}$ The $2 \mathrm{H} \rightarrow 1 \mathrm{~T}$ transformation is accompanied by changes in coordination from trigonal prismatic to octahedral and metal-metal bond distortions. ${ }^{27,29,32,42}$ However, one outstanding issue of these studies was the lack of understanding of whether these effects were 

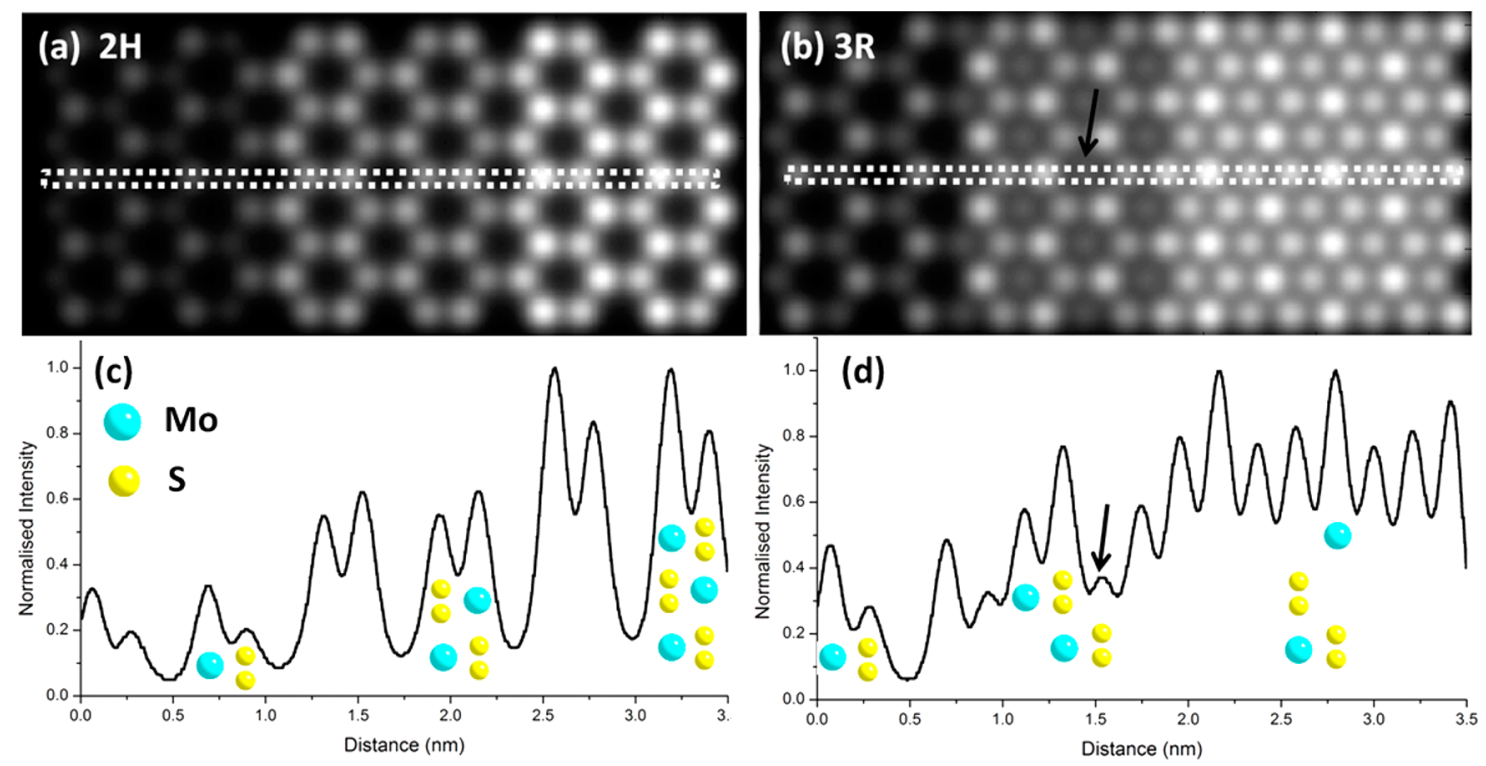

Figure 2. ADF STEM simulations of the (a) $2 \mathrm{H} \mathrm{MoS}_{2}$ structure shown in Figure 1a and (b) $3 \mathrm{R} \mathrm{MoS}$ structure shown in Figure $1 \mathrm{~b}$. $(c, d)$ Corresponding intensity profiles. The arrows in (b) and (c) indicate faint signal from sulfur columns in the bilayer region.

intrinsically due to exfoliation or to the presence of intercalated guest molecules among the layers. $\mathrm{MoS}_{2}$ composites prepared via lithium intercalation indicated the retained presence of lithium in the restacked material even after washing. ${ }^{43}$ This, in turn, undermines the claim that $1 \mathrm{~T}$ phase is metastable even after removal of $\mathrm{Li}^{27}$

Our findings on liquid-phase exfoliated TMDs show that deviations in the stacking sequence can indeed exist. The observed nonbulk stacking sequences can be obtained by applying simple translational shifts to the layers of the bulk structures and are not accompanied by changes in the coordination of the metal ion. This is expected to ultimately alter the charge capacity when these materials are used in batteries and supercapacitors and the reactivity when they are used in catalysis or gas sensing. The electronic and optical properties of these materials are also expected to be altered by nonbulk stacking deviations, having strong impact on the ultimate commercial integration of these materials.

\section{RESULTS}

ADF STEM provides directly interpretable images, where one expects the intensity to be dominated by the heavier atoms and to scale linearly with the number of layers. Furthermore, thanks to the Z-contrast mechanism, it is possible to distinguish atomic columns containing $\mathrm{Mo}^{4+}(Z=42) / \mathrm{W}^{4+}(Z=74)$ ions from those with $S^{2-}(Z=16)$ ions when observed in the [001] direction, perpendicular to the basal planes.

There is, however, always a possibility that the strong signal arising from atomic columns containing heavier metal ions can make it difficult to observe the signal of the nearest, much lighter sulfur columns. The image pattern ultimately generated is expected to depend heavily on the stacking sequence and the number of layers.

In the $2 \mathrm{H}$ polytype (Figure 1a), the projected pattern will depend on the number of layers: monolayers will produce a simple hexagonal pattern with a single atomic column around each of the 2D Bravais lattice point, while multilayers will produce a honeycomb pattern with two atomic columns around each of the 2D Bravais lattice point. The lattice spacingis 3.16 and $3.15 \AA$ for $\mathrm{MoS}_{2}$ and $\mathrm{WS}_{2}$, respectively.

In the $3 \mathrm{R}$ polytype (Figure $1 \mathrm{~b}$ ), the $\mathrm{Mo}^{4+} / \mathrm{W}^{4+}$ ions in the monolayer and bilayer regions will form hexagonal and honeycomb patterns, respectively, similarly to the $2 \mathrm{H}$ polytype. However, the trilayers will form hexagonal patterns with much smaller lattice spacing (1.83 $\AA$ for $\mathrm{MoS}_{2}$ and $1.82 \AA$ for $\mathrm{WS}_{2}$ ). It must be also noted that, while the patterns formed in the bilayer regions are similar for both $2 \mathrm{H}$ and $3 \mathrm{R}$ polytypes, the actual structures are fundamentally different.

The above discussion assumes that liquid-phase exfoliated samples do not undergo structural changes within the layers. This is confirmed by the analysis of the high-resolution transmission electron microscopy images (Figure S1) presented in the Supporting Information (SI).

$\mathrm{MoS}_{2}$ Nanosheets. ADF STEM image simulations were computed on the bases of the structural models in Figure 1 to support the discussions above. These are shown in Figure 2. As expected, the image intensity is dominated by the presence of $\mathrm{Mo}^{4+}$ columns: in the $2 \mathrm{H}$ polytype, we assist a proportional increase of intensities with each additional layer (Figure $2 \mathrm{c}$ ). The situation is more complicated for the $3 \mathrm{R}$ polytype because certain columns in the bilayer and trilayer regions have 


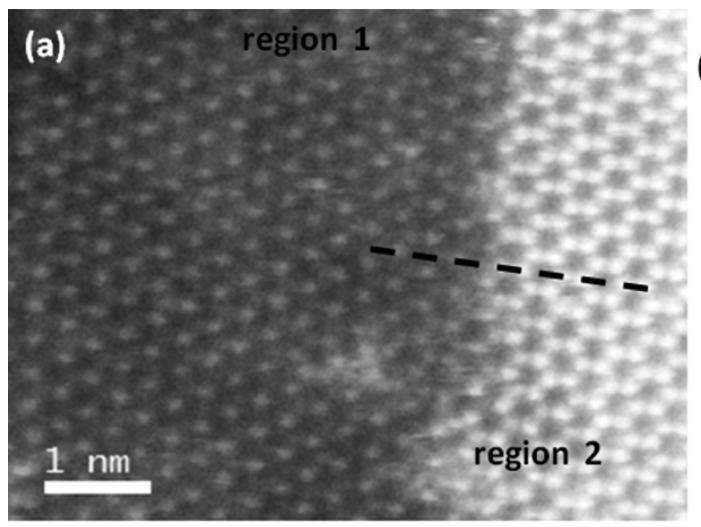

(b)

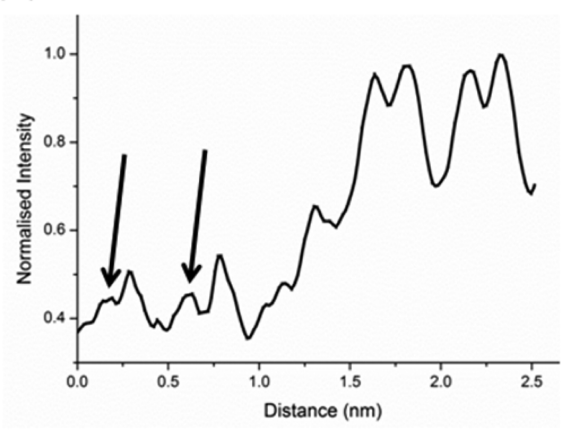

Figure 3. (a) ADF STEM image of a typical $\mathrm{MoS}_{2}$ nanosheet with (b) intensity profile taken along the dotted line. The original image included a much larger field of view and was cropped so that the vacuum region is no longer visible.

the same number of equivalent Mo and S atoms, therefore generating similar peak intensities.

The experimental images agree well with these discussions. Figure 3a shows an image where both hexagonal (region 1) and honeycomb (region 2) patterns are readily identifiable, with lattice spacing of 3.16 $\AA$. On the basis of the above analysis, it can be concluded that this sheet is consistent with a $2 \mathrm{H}$ polytype, with region 1 being a monolayer and region 2 being a multilayer.

In the monolayer region, the intensity arising from the $\mathrm{Mo}^{4+}$ ions partially suppresses the signal from the $\mathrm{S}^{2-}$; nonetheless, the position of sulfur columns remains detectable as evidenced by the arrows in the intensity profile (Figure 3b).

The peak image intensity doubles across the step into the multilayer region, corresponding either to a one-to-two, two-to-four, or three-to-six layer steps. Knowing that region 1 is a monolayer is sufficient information to establish that region 2 is indeed a bilayer.

For 3R bilayer sheets, simulations predict a weak signal arising from the sulfur columns in the middle of honeycombs (marked with arrows in Figure 2b,d). Such signal is not observed in the bilayer region in Figure 3, implying that this nanosheet has $2 \mathrm{H}$ structure. Unfortunately, in the experimental images, such faint sulfur signal is most likely obscured by the nearby columns. Nonetheless, simulations of 3R polytype bilayer regions evidence a noticeable intensity difference between nearest neighbor columns. This is expected because one column has only a single $\mathrm{Mo}^{4+}$ ion while the second column has one $\mathrm{Mo}^{4+}$ and two $\mathrm{S}^{2-}$ ions. This difference is much smaller for the $2 \mathrm{H}$ polytype bilayer (simulation) where nearest neighbor columns have the same numbers of equivalent ions. Hence, it can be concluded that the image in Figure $3 a$ is indeed of $2 \mathrm{H}$ polytype. Moreover, unlike the simulation (Figure 2a), the experimental image of the bilayer region in Figure $3 a$ does not show noticeable difference between nearest neighbor columns. This

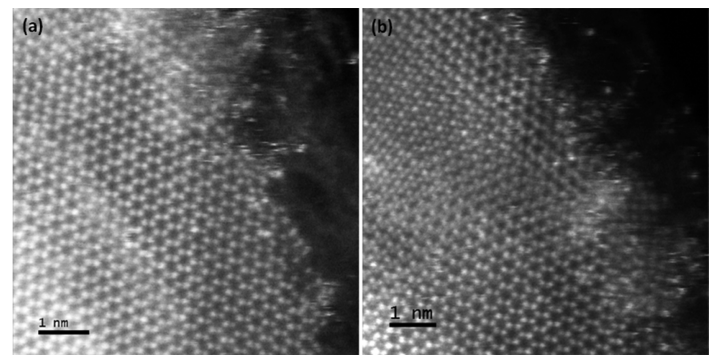

Figure 4. (a,b) ADF STEM images of $\mathrm{MoS}_{2}$ nanosheets.

mismatch might be due to the fact that accurate values of the Debye-Waller factors for Mo and S atoms are not known.

The $2 \mathrm{H}$ and $3 \mathrm{R}$ polytypes can be reliably and quantitatively distinguished by simple analysis of the projected patterns when sheets are $\geq 3$ layers thick. An example is given in Figure 4. In Figure 4a, the atomic columns form a honeycomb pattern throughout the entire image, confirming a $2 \mathrm{H}$ polytype. In Figure $4 \mathrm{~b}$, the pattern deviates from the honeycomb as we move from the bottom to the top-left corner of the image, where additional atomic columns appear in the center of each honeycomb, with formation of a hexagonal pattern with $1.83 \AA$ lattice spacing. This projection is consistent with a $3 \mathrm{R}$ polytype.

An entirely different stacking sequence was however observed in a few cases. In Figure 5a, we show an image of a nanosheet where a hexagonal pattern with $3.16 \AA$ spacing is observable throughout the entire field of view. The intensity profile taken across the flake (black dotted line) reveals the presence of a step, with intensity doubling across the image, which indicates that monolayer and bilayer regions are both present. The sole explanation for the hexagonal pattern to be maintained throughout the image, both in the monolayer and the bilayer regions, is that the Mo atoms of the second layer are stacked on top of the Mo atoms belonging to the first layer (homonuclear stacking).

The position of the $\mathrm{S}$ atoms in this image must be identified in order to propose an appropriate structural 
model. The intensity profile relative to the monolayer region (taken across the white dotted line) shows diffuse $S$ peaks in the expected positions (see arrows in Figure $5 c$ ). In the bilayer region noise, probe tail effects, and off-axis tilts are magnified, hiding the $S$ atoms entirely as a result.

The inability to identify the sulfur positions in the bilayer region results in two possible models, which can consistently explain the multilayer region in Figure 5. These can be simply derived from the $2 \mathrm{H}$ and $3 \mathrm{R}$ polytypes, respectively, by shifting the second layer of one atomic plane along the vectors $\mathbf{R}$ or $\mathbf{R}^{*}$ as shown in Figure 6 . The new structures can be referred as $1 \mathrm{H}$ and
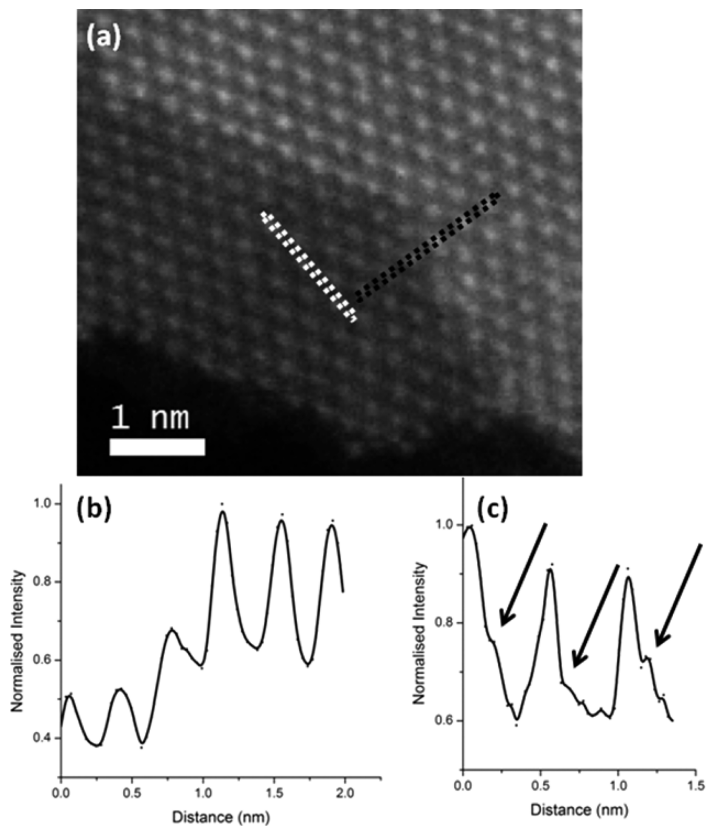

Figure 5. (a) ADF STEM image of a $\mathrm{MoS}_{2}$ nanoflake. (b) Intensity profile taken along the black dotted line. (c) Intensity profile taken along the white dotted line.
$2 \mathrm{H}^{\prime}$ respectively. As indicated in Figures $6 \mathrm{c}, \mathrm{d}$, Mo atoms are stacked on the top of each other (homonuclear stacking) in both models. The ADF STEM image simulations of these polytypes are given in the SI (Figure S2).

The possibility that sample tilt can lead to an apparent nonbulk stacking was also investigated. It was determined that rotation of the nanoflake by $30.7^{\circ}$ is required to obtain a projected structure somewhat similar to the homonuclear stacking (SI, Figure S3). However, it is only limited to the bilayer region. More importantly, this results in a $\sim 10 \%$ apparent reduction of the lattice spacing (from 3.16 to $2.84 \AA$ ). Hence, it can be concluded that the observed patterns are indeed due to a homonuclear stacking and not to inadvertent sample tilt.

Although the mechanism for formation of the nonbulk phases is debatable, it is believed that layers can glide along the vectors $\mathbf{R}$ or $\mathbf{R}^{*}$ (or equivalent directions) during the exfoliation process. The possibility that these stacking sequences are formed by random restacking upon deposition is less plausible as it would imply that complete exfoliation is achieved by liquid-phase dispersion. Unfortunately, there is no evidence to substantiate such a claim since turbostratic stacking was not observed in few-layer sheets. Nonetheless, in four cases, an orientational mismatch corresponding to $6 \pm 1^{\circ}$ in-plane rotation was observed. In all these cases, the nanoflakes appeared contaminated with impurities (SI, Figure S4). The presence of contaminants is thought to be responsible for the smallangle in-plane rotation.

From the 27 different $\mathrm{MoS}_{2}$ nanoflakes imaged and analyzed, 17 were found to consist of a $2 \mathrm{H}$ polytype, nine with a homonuclear stacking, and only one with a 3R polytype. A single case where the nanoflake displayed both homonuclear and $2 \mathrm{H}$ stacking was also observed (SI, Figure S5). (a) $2 \mathrm{H}$

[001]

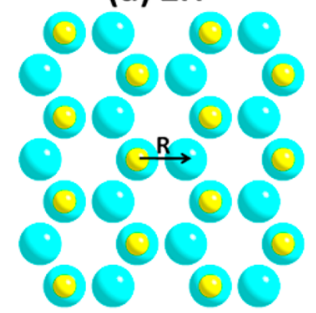

[110]

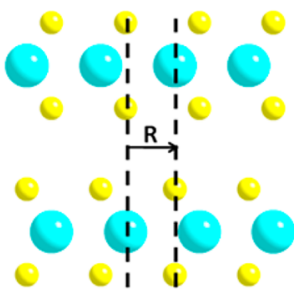

(b) $3 R$
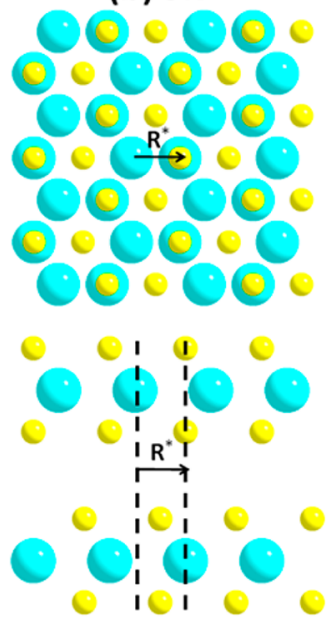

(c) $2 \mathrm{H}^{\prime}$

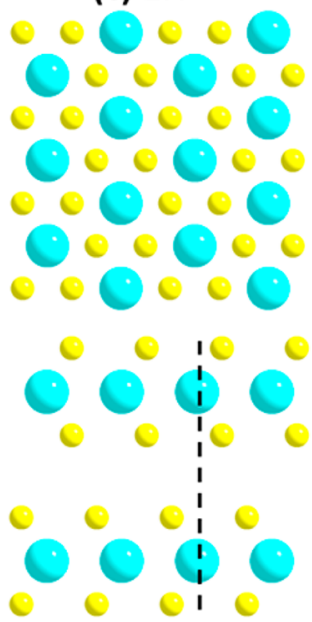

(d) $1 \mathrm{H}$

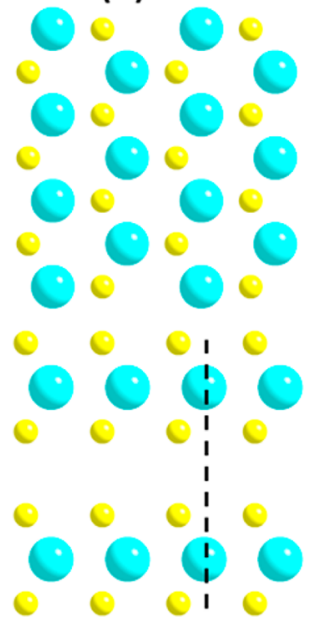

Figure 6. Second layers of the (a) $2 \mathrm{H}$ and (b) $3 \mathrm{R}$ polytypes are shifted, respectively, along the $\mathrm{R}$ and $\mathrm{R}^{*}$ vectors in order to obtain homonuclearly stacked (c) $2 \mathrm{H}^{\prime}$ and (d) $1 \mathrm{H}$ structures. 

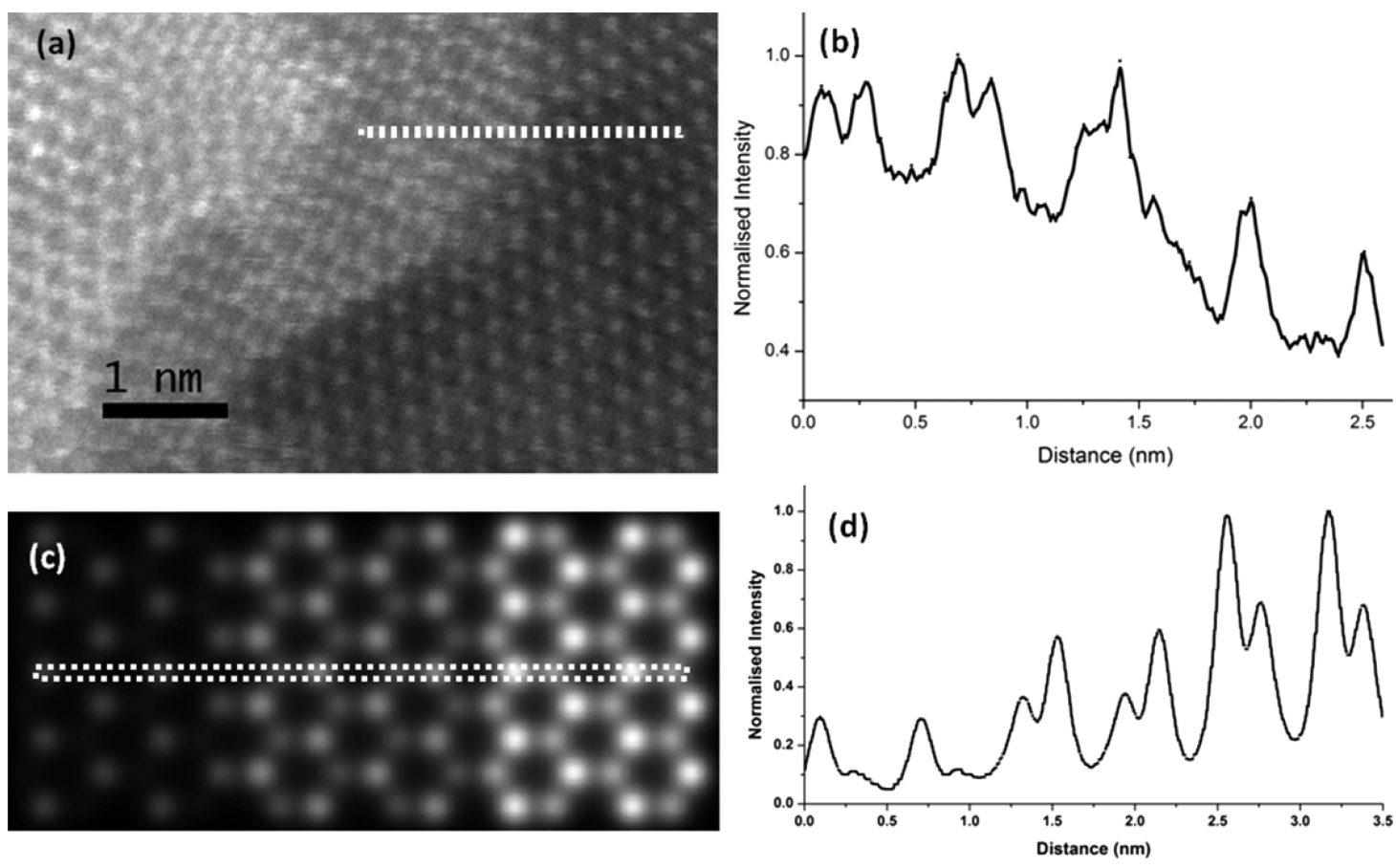

Figure 7. (a) ADF STEM image of a $\mathrm{WS}_{2}$ nanosheet with corresponding (b) intensity profile drawn across the white dotted line. The image in (a) was originally cropped to remove the vacuum from the field of view. (c) ADF image simulation of the $\mathrm{WS}_{2}$ structure shown in Figure 1a with (d) corresponding intensity profile.

In addition to the evidence of unusual crystal stacking in liquid-phase exfoliated few-layer $\mathrm{MoS}_{2}$ nanosheets, one interesting finding came from the quantitative analysis of the $3 R$ polytype sheet (Figure 2d). Careful analysis in this case revealed some lattice distortions beyond scan noise. The shear strain was calculated to be between 11 and 26\% (100\% was considered to be the value of the shear strain required to shift one single layer to produce a $1 \mathrm{H}$ from a $3 R$ polytype in a bilayer configuration, Figure $6 b$ ). A magnified view of a region of Figure $2 \mathrm{~d}$ and details of shear strain calculations are given in the $\mathrm{Sl}$, Figures S6-S8.

WS $_{2}$ Nanosheets. A similar structural study was extended to $\mathrm{WS}_{2}$ nanosheets with the purpose to see whether the unusual stacking sequence obtained for liquid-phase exfoliated $\mathrm{MoS}_{2}$ was consistently found for other similarly exfoliated, isostructural TMDs.

In Figure 7a, we show an ADF STEM image of a WS ${ }_{2}$ nanosheet consistent with the $2 \mathrm{H}$ polytype. Similarly to $\mathrm{MoS}_{2}$, the monolayer region has a hexagonal pattern with $3.15 \AA$ spacing, while the multilayer region shows a honeycomb pattern. Counting the number of layers can once again be confirmed by analysis of the intensity profile across the sheet, revealing a step transition between a monolayer and bilayer (Figure 7b).

The strong contrast originating from high- $Z \mathrm{~W}$ atoms $(Z=74)$ makes it difficult to observe much lighter atoms like $S(Z=16)$. This makes the $S$ atoms in monolayers completely disappear next to the much heavier W neighbor atoms.

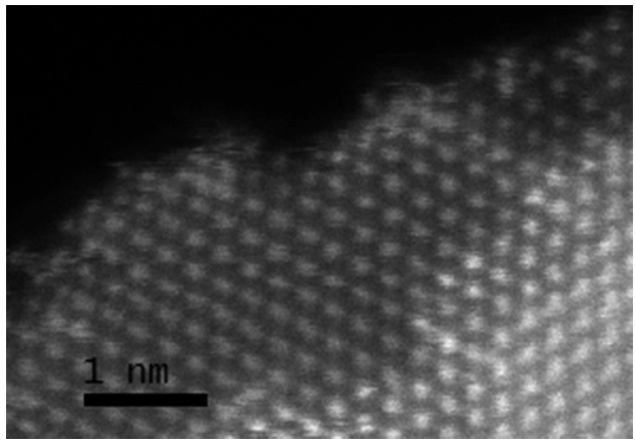

Figure 8. ADF STEM image of a $W_{2}$ nanoflake.

ADF simulations were carried out to corroborate our image interpretation. Since $W S_{2}$ is isostructural to $M_{2}$, we used the same model as in Figure 1a, with the appropriate modification of the lattice spacing. The simulation results are shown in Figure $7 c$, together with the relative intensity profile across the step (Figure 7d). As expected, the intensity on the monolayer region is dominated by a very strong signal generated by the $\mathrm{W}$ atoms, while the $S$ atoms are almost completely hidden.

As in the case of liquid-phase exfoliated $\mathrm{MoS}_{2}$ flakes, $\mathrm{WS}_{2}$ did not always provide sole evidence for $2 \mathrm{H}$ polytype. Figure 8 shows a stepped nanoflake where the hexagonal pattern is preserved over the whole field of view. Once again, the only explanation can be that the $W$ atoms of each consecutive layer are stacked homonuclearly on the top of the $\mathrm{W}$ atoms of the previous layer. The two possible models are the $2 \mathrm{H}^{\prime}$ and the $1 \mathrm{H}$ described in Figure 6. 
Finally, the position of the $S$ atoms in the protruding monolayer in Figure $7 \mathrm{a}$ can be determined by careful statistical analysis on the ADF intensities. W atoms form hexagonal structure with two equivalent sites " $B$ " and " $C$ " (Figure S9), which can be occupied by the $S$ atoms. The calculated normalized averaged intensities at the " $\mathrm{B}$ " and " $\mathrm{C}$ " sites in experimental image (Figure 7a) are $1.00 \pm 0.010$ and $0.959 \pm 0.012$, respectively. The difference is small but nevertheless statistically distinguishable and significant. Furthermore, the positions of the $S$ columns might be accurately determined by simultaneous acquisition of ADF and annular brightfield ( $A B F)$ signals. The missing sulfur columns are in fact expected to be resolved in the ABF image.

Recently, several detailed studies on radiation damage at $80 \mathrm{keV}^{36,37}$ (TEM) and $60 \mathrm{keV}^{38}$ (STEM) in monolayer $\mathrm{MoS}_{2}$ have been published, reporting noticeable damage to the monolayer region over time. In this work (80/60 keV STEM), the electron beam damage was negligible and did not hinder the imaging process. However, our samples are mainly few-layer Mo/WS 2 with small area protruding monolayers. For such samples, electron damage is expected to be weakened. In a single case, for large protruding $\mathrm{WS}_{2}$ monolayer, strong damage was observed (Figure S10).

Overall, from the five different $\mathrm{WS}_{2}$ nanosheets imaged and analyzed, only one was consistent with the $2 \mathrm{H}$ polytype, while all the others possessed homonuclear stacking. The 3R polytype was never observed. None of the images displayed relative in-plane rotation of the layers.

Electronic Structure. In order to validate the STEM analysis and to assess the relative structural stability of the various polytypes, we have performed first principles electronic structural calculations based on density functional theory. In particular, we have considered both the standard local density approximation (LDA) and the van der Waals corrected exchange and correlation functional of Tkatchenko and Scheffler $(T S-v d W)^{44}$ (see Experimental Methods section for more details). In Figure 9, we present the interlayer binding energy (per unit area) for the various stacks as a function of the interlayer distance, $d$.

It is clear from the figure that the binding energies of the $2 \mathrm{H}$ and $3 \mathrm{R}$ geometries are essentially identical, indicating that the two stacks are almost equally energetically favorable. Rotational transformation from the $2 \mathrm{H}$ into the $3 \mathrm{R}$ structure is inhibited by a potential barrier. This is computed with the nudged elastic band method in the upper inset of Figure 9, where we show the total energy (per unit cell) profile along the rotation, bringing the $2 \mathrm{H}$ structure onto the $3 \mathrm{R}$. Clearly, there is a significant energy barrier for the rotation, which explains near complete absence of the rotational disorder in the nanoflakes.

Note that any other geometry presents significant smaller binding energy and that the binding energy is

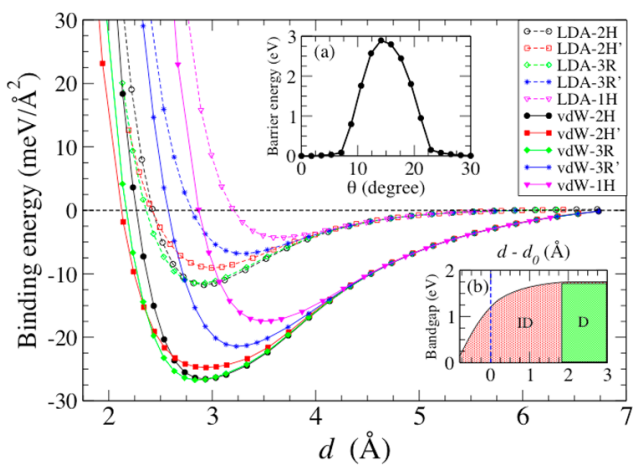

Figure 9. Binding energy density as a function of the interlayer distance for $\mathrm{MoS}_{2}$ bilayers presenting the stacks investigated. Results are for both the LDA (dashed lines) and the TS-van der Waals corrected (solid lines) functionals. Note that the two functionals return rather similar equilibrium binding distance, but different binding energies. Note also that the binding energies for the $2 \mathrm{H}$ and 3R stack are almost degenerate. In (a), we show the barrier energy profile for rotating the $2 \mathrm{H}$ structure into the $3 \mathrm{R}$ one. Each step in the rotation path is characterized by the planar rotation angle, $\theta$. In (b), the variation of the LDA band gap (calculated without spin-orbit coupling) is plotted against the layer separation, $d$, for a $2 \mathrm{H}$ polytype $\mathrm{MoS}_{2}$ bilayer; $d_{0}$ is the equilibrium distance. In the figure, we also report results for a new hypothetical structure named $3 R^{\prime}$. This is obtained by applying the same translation that transforms $3 \mathrm{R}$ in $1 \mathrm{H}$, but by only $1 / 2$ of the translational lattice vector.

essentially a function of the interlayer distance as shown in Figure 9. Next we look at the electronic structure. The lower inset of Figure 9 reports the bilayer band gap for the $2 \mathrm{H}$ stack (calculated at the LDA level without including spin-orbit interaction) as a function of the interlayer distance, $d\left(d_{0}\right.$ is the equilibrium binding distance). At equilibrium, the bilayer displays an indirect band gap, which remains indirect for any compressions and for elongations of $d$, up to about $2 \AA$ above the equilibrium binding distance. For extremely compressed interlayer distances, the gap reduces drastically, while for $\left(d-d_{0}\right)>2 \AA$, the direct gap characteristic of the single-layer limit is approached.

Finally, in Figure 10, we present the electronic band structure of the various stacks, calculated at the relative equilibrium distance and by considering spin-orbit interaction. For all cases, the valence band maximum moves from the $\mathrm{K}$ point for a $\mathrm{MoS}_{2}$ monolayer to the $\Gamma$ point in the case of the bilayers. Kramer's degeneracy is always satisfied at $\Gamma$ so that no band-spin-splitting is present. In contrast, the conduction band maximum is along the $\Gamma-\mathrm{K}$ line, and when inversion symmetry is broken, the bands spin-split (Figure 10e). Such a situation is encountered for instance in the case of the $3 \mathrm{R}$ and $1 \mathrm{H}$ stack, the second and third most abundant configurations found after exfoliation. This is a rather intriguing result. In fact, when going from $\mathrm{MoS}_{2}$ monolayers to bilayers with $3 \mathrm{R}$ or $1 \mathrm{H}$ symmetry, one transfers the spin-polarization from the valence band maximum to the conduction band minimum. As such, the $3 \mathrm{R} / 1 \mathrm{H}$ bilayers can be used as conduction band spintronics 


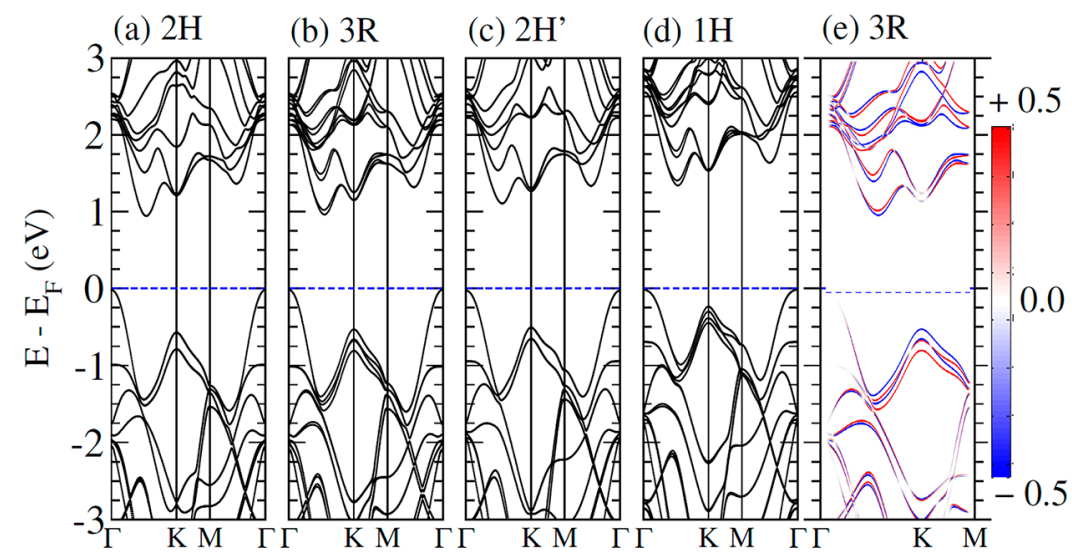

Figure 10. Electronic band structure calculated at the LDA level and including spin-orbit interaction for the various stacks investigated. Note that the valence band maximum for all the stacks is now at the $\Gamma$ point and is not spin-polarized. In contrast, the conduction band minimum is midway along the $\Gamma-K$ line, and in the case of stacks presenting broken inversion symmetry, it is spin-polarized. The last panel displays the spin-projection of the bands for the $3 R$ stack: red and blue lines represent, respectively, spin-up and spin-down projections.

materials in contrast to monolayers, which are spinpolarized in the valence band. ${ }^{45}$ This result opens up the possibility of using $\mathrm{MoS}_{2}$ bilayers as a $2 \mathrm{D}$ electronbased materials platform for valleytronics. ${ }^{46}$ Note that recently valleytronics was reported for $2 \mathrm{H}$-stacked bilayers in which the symmetry is broken by a transverse electric field. ${ }^{47}$ In the case presented here, the symmetry breaking is spontaneous; namely, it is a direct consequence of the layer stack obtained by exfoliation. A similar result has also been obtained for $\mathrm{WS}_{2}$ (see $\mathrm{SI}$ ).

\section{CONCLUSIONS}

In this work, we carried out imaging and structural characterization of 2D TMD nanostructures $\left(\mathrm{MoS}_{2}\right.$ and $W_{2}$ ) produced by liquid-phase exfoliation. On the basis of ADF STEM and image simulations, it was determined that nonbulk stacking, here referred as $1 \mathrm{H}$ and $2 \mathrm{H}^{\prime}$ polytypes, can exist. This previously unreported homonuclear stacking consists of metal ions placed exactly on top of each other for each consecutive layer. Twenty-seven different $\mathrm{MoS}_{2}$ nanoflakes were imaged and analyzed. Among these, 17 were consistent with a $2 \mathrm{H}$ polytype, nine with a homonuclear stacking, and only one with a 3R polytype. Moreover, a nanoflake displaying both homonuclear and $2 \mathrm{H}$ stacking was also observed. The nanoflake in 3R polytype configuration exhibited a significantly strained lattice. Among the five $\mathrm{WS}_{2}$ nanoflakes examined, one exhibited a $2 \mathrm{H}$ structure, while all the others were consistent with homonuclear stacking. The $3 \mathrm{R}$ polytype was never observed for $\mathrm{WS}_{2}$.

Electronic structure calculations for such bilayer stacks reveal that $2 \mathrm{H}$ and $3 \mathrm{R}$ polytypes are almost energetically degenerate and that they can hardly be transformed from one into the other by lattice rotation. Most intriguingly, the band structure analysis shows that the valence band maximum is always at the $\Gamma$ point and as such is not spin-polarized. In contrast, the conduction band minimum is located along the $\Gamma-\mathrm{K}$ line and presents spin-polarization for those stacks with broken inversion symmetry. As a consequence, our results support the idea that $\mathrm{MoS}_{2}$ and $\mathrm{WS}_{2}$ bilayers can be used as electron conductive spintronics materials.

\section{EXPERIMENTAL METHODS}

$\mathrm{MoS}_{2}$ or $\mathrm{WS}_{2}$ dispersions were produced by ultrasonication (UP 100H Ultrasonic Processor, Hielscher, 100\% amplitude, continuous cycle) of as-purchased raw powders in $\mathrm{N}$-methylpyrrolidone (NMP) for $2 \mathrm{~h}$. During ultrasonication, dispersions were placed in an ice bath to minimize heating and relative temperature effects. After ultrasonication, the dispersions were left to settle down for two nights. Finally, the supernatant was carefully decanted, and TEM samples were drop-casted onto holey carbon/copper grids ( 500 mesh). The samples were baked for $3 \mathrm{~h}$ at $135^{\circ} \mathrm{C}$ in vacuum.

$\mathrm{MoS}_{2}, \mathrm{WS}_{2}$ powders, and NMP were purchased from SigmaAldrich and used as received (product nos. 234842, 243639, and 328634 , respectively). ADF STEM imaging of $\mathrm{MoS}_{2}$ nanoflakes was performed using a JEM-ARM200F aberration-corrected STEM microscope operated at $80 \mathrm{keV}$ (probe current of $\sim 34 \mathrm{pA}$, with a probe convergence angle of $22 \mathrm{mrad}$ and a collection half angle of 55-170 mrads). ADF STEM imaging of $\mathrm{MoS}_{2}$ and $\mathrm{WS}_{2}$ nanoflakes was performed using a Nion UltraSTEM100 aberration-corrected STEM microscope operated at $60 \mathrm{keV}$ (probe current of $\sim 50 \mathrm{pA}$, probe convergence angle of 30 mrad, and collection half angle of 78-195 mrads). HRTEM images were acquired on the double aberration-corrected JEOL $2200 \mathrm{MCO}$. The instrument was operated at $80 \mathrm{keV}$ with spherical aberration set to $C_{3}=-3 \mu \mathrm{m}$. All experimental images were filtered using Butterworth filter which removed high-frequency noise. All simulations were performed using the QSTEM code, ${ }^{48}$ which is based on the frozen phonon approach. The number of runs was set to 20. For the simulations of $\mathrm{MoS}_{2}$ nanoflakes, ARM200F microscope parameters were used. For the simulations of $\mathrm{WS}_{2}$ nanoflakes, Nion UltraSTEM100 microscope parameters were used.

We have performed the first-principle calculations based on both local density approximated (LDA) and van der Waals (vdW) 
corrected density functional theory (DFT), as implemented in the all-electron, numerical basis code FHI-AIMS. ${ }^{49}$ For vdW-DFT calculation, the generalized gradient approximation of Perdew, Burke, and Ernzerhof $(\mathrm{PBE})^{50}$ augmented with the TkatchenkoScheffler-vdW (TS-vdW) correction ${ }^{44,51}$ is employed. The "firsttier" basis set composed of atomic-centered numeric orbitals are used. Brillouin zone sampling is done by using a $(12 \times$ $12 \times 1)$ Monkhorst-Pack k-grid. Periodic boundary conditions are applied, and a vacuum layer of at least $15 \AA$ is placed above the bilayers or monolayer to minimize the interaction between periodic images. The conjugate gradient method is used to obtain relaxed geometries. Both atomic positions and cell parameters are allowed to relax until the forces on each atom are less than $0.01 \mathrm{eV} / \AA ̊ \AA$. We also calculate the electronic structure using LDA-DFT approach with the inclusion of spin-orbit (SO) interaction via onsite approximation, ${ }^{52}$ as implemented in the numerical basis code SIESTA. ${ }^{53}$ The climbing image nudged elastic band (CI-NEB) method implemented in the DFT code SIESTA ${ }^{54}$ is used to determine the minimum energy pathway of the energy barrier between the two different stacked sequences in bilayer $\mathrm{MoS}_{2}$ (i.e., $2 \mathrm{H}$ and $3 \mathrm{R}$ ).

Conflict of Interest: The authors declare no competing financial interest.

Acknowledgment. V.N. wishes to knowledge support from the European Research Council (ERC Starting Grant 2DNanoCaps) and Science Foundation Ireland. S.S. and K.D. wish to thank funding from CRANN. Computational resources have been provided by the Trinity Centre for High Performance Computing (TCHPC). The authors also wish to thank the Advanced Microscopy Laboratory and SuperSTEM for access to their facilities.

Supporting Information Available: Analysis of the HRTEM images; simulations of the homonuclear stacking sequences; effect of rotation on the projected pattern; minor rotational disorder in $\mathrm{MoS}_{2}$ nanoflakes; image of the nanoflake displaying homonuclear and distorted $2 \mathrm{H}$ stacking; shear strain calculations; schematic of $2 \mathrm{D}$ hexagonal unit cell displaying site to accommodate S columns; $2 \mathrm{D} \mathrm{WS}_{2}$ time series; electronic structure. This material is available free of charge via the Internet at http://pubs.acs.org.

\section{REFERENCES AND NOTES}

1. Nicolosi, V.; Chhowalla, M.; Kanatzidis, M. G.; Strano, M. S.; Coleman, J. N. Liquid Exfoliation of Layered Materials. Science 2013, 340, 1226419.

2. Geim, A. K.; Grigorieva, I. V. van der Waals Heterostructures. Nature 2013, 499, 419-425.

3. Radisavljevic, B.; Radenovic, A.; Brivio, J.; Giacometti, V.; Kis, A. Single-Layer $\mathrm{MoS}_{2}$ Transistors. Nat. Nanotechnol. 2011, 6, 147-150.

4. Kim, K. S.; Zhao, Y.; Jang, H.; Lee, S. Y.; Kim, J. M.; Kim, K. S.; Ahn, J.-H.; Kim, P.; Choi, J.-Y.; Hong, B. H. Large-Scale Pattern Growth of Graphene Films for Stretchable Transparent Electrodes. Nature 2009, 457, 706-710.

5. Georgiou, T.; Jalil, R.; Belle, B. D.; Britnell, L.; Gorbachev, R. V.; Morozov, S. V.; Kim, Y.-J.; Gholinia, A.; Haigh, S. J.; Makarovsky, O.; et al. Vertical Field-Effect Transistor Based on Graphene-WS $\mathrm{W}_{2}$ Heterostructures for Flexible and Transparent Electronics. Nat. Nanotechnol. 2012, 7, 4-7.

6. Schedin, F.; Geim, A. K.; Morozov, S. V.; Hill, E. W.; Blake, P.; Katsnelson, M. I.; Novoselov, K. S. Detection of Individual Gas Molecules Adsorbed on Graphene. Nat. Mater. 2007, 6, 652-655.

7. Li, H.; Yin, Z.; He, Q.; Li, H.; Huang, X.; Lu, G.; Fam, D. W. H.; Tok, A. I. Y.; Zhang, Q.; Zhang, H. Fabrication of Single- and Multilayer $\mathrm{MoS}_{2}$ Film-Based Field-Effect Transistors for Sensing NO at Room Temperature. Small 2012, 8, 63-67.

8. Tye, C. T.; Smith, K. J. Catalytic Activity of Exfoliated $\mathrm{MoS}_{2}$ in Hydrodesulfurization, Hydrodenitrogenation and Hydrogenation Reactions. Top. Catal. 2006, 37, 129-135.

9. Gourmelon, E. $\mathrm{MS}_{2}(\mathrm{M}=\mathrm{W}, \mathrm{Mo})$ Photosensitive Thin Films for Solar Cells. Sol. Energy Mater. Sol. Cells 1997, 46, 115-121.
10. Frame, F. A.; Osterloh, F. E. CdSe-MoS 2 : A Quantum SizeConfined Photocatalyst for Hydrogen Evolution from Water under Visible Light. J. Phys. Chem. C 2010, 114, 10628-10633.

11. Soon, J. M.; Loh, K. P. Electrochemical Double-Layer Capacitance of $\mathrm{MoS}_{2}$ Nanowall Films. Electrochem. Solid-State Lett. 2007, 10, A250-A254.

12. Du, G.; Guo, Z.; Wang, S.; Zeng, R.; Chen, Z.; Liu, H. Superior Stability and High Capacity of Restacked Molybdenum Disulfide as Anode Material for Lithium Ion Batteries. Chem. Commun. 2010, 46, 1106-1108.

13. Dutta, P. K.; Auerbach, S. M.; Carrad, K. A. Handbook of Layered Materials; CRC Press: Boca Raton, FL, 2004.

14. Schlenker, C.; Dumas, J.; Greenblatt, M.; van Smaalen, S. Physics and Chemistry of Low-Dimensional Inorganic Conductors; Plenum Press: New York, 1996.

15. Allan, D. R.; Kelsey, A. A.; Clark, S. J.; Angel, R. J.; Ackland, G. J. High-Pressure Semiconductor-Semimetal Transition in $\mathrm{TiS}_{2}$. Phys. Rev. B 1998, 57, 5106-5110.

16. Kam, K. K.; Parkinson, B. A. Detailed Photocurrent Spectroscopy of the Semiconducting Group VI Transition Metal Dichalcogenides. J. Phys. Chem. 1982, 86, 463-467.

17. Frindt, R. F.; Yoffe, A. D. Physical Properties of Layer Structures: Optical Properties and Photoconductivity of Thin Crystals of Molybdenum Disulphide. Proc. R. Soc. London, Ser. A 1963, 273, 69-83.

18. Frindt, R. Optical Absorption of a Few Unit-Cell Layers of $\mathrm{MoS}_{2}$. Phys. Rev. 1965, 140, A536-A539.

19. Miremadi, B. K.; Morrison, S. R. The Intercalation and Exfoliation of Tungsten Disulfide. J. Appl. Phys. 1988, 63, 4970-4974.

20. Splendiani, A.; Sun, L.; Zhang, Y.; Li, T.; Kim, J.; Chim, C. Emerging Photoluminescence in Monolayer $\mathrm{MoS}_{2}$. Nano Lett. 2010, 10, 1271-1275.

21. Mak, K.; Lee, C.; Hone, J.; Shan, J.; Heinz, T. Atomically Thin $\mathrm{MoS}_{2}$ : A New Direct-Gap Semiconductor. Phys. Rev. Lett. 2010, 105, 2-5.

22. Zhao, W.; Ghorannevis, Z.; Chu, L.; Toh, M.; Kloc, C.; Tan, P.-H.; Eda, G. Evolution of Electronic Structure in Atomically Thin Sheets of $\mathrm{WS}_{2}$ and WSe $\mathrm{W}_{2}$. ACS Nano 2013, 7, 791-797.

23. Joensen, P.; Frindt, R.; Morrison, S. Single-Layer $\mathrm{MoS}_{2}$. Mater. Res. Bull. 1986, 21, 457-461.

24. Coleman, J. N.; Lotya, M.; O'Neill, A.; Bergin, S. D.; King, P. J.; Khan, U.; Young, K.; Gaucher, A.; De, S.; Smith, R. J.; et al. Two-Dimensional Nanosheets Produced by Liquid Exfoliation of Layered Materials. Science 2011, 331, 568-571.

25. Smith, R. J.; King, P. J.; Lotya, M.; Wirtz, C.; Khan, U.; De, S.; O'Neill, A.; Duesberg, G. S.; Grunlan, J. C.; Moriarty, G.; et al. Large-Scale Exfoliation of Inorganic Layered Compounds in Aqueous Surfactant Solutions. Adv. Mater. 2011, 23, 3944-3948.

26. Liang, Y.; Feng, R.; Yang, S.; Ma, H.; Liang, J.; Chen, J. Rechargeable Mg Batteries with Graphene-like $\mathrm{MoS}_{2}$ Cathode and Ultrasmall Mg Nanoparticle Anode. Adv. Mater. 2011, 23, 640-643.

27. Yang, D.; Sandoval, S.; Divigalpitiya, W.; Irwin, J.; Frindt, R. Structure of Single-Molecular-Layer $\mathrm{MoS}_{2}$. Phys. Rev. B 1991, 43, 12053-12056.

28. Lee, C.; Yan, H.; Brus, L. E.; Heinz, T. F.; Hone, J.; Ryu, S. Anomalous Lattice Vibrations of Single- and Few-Layer $\mathrm{MoS}_{2}$. ACS Nano 2010, 4, 2695-2700.

29. Gordon, R.; Yang, D.; Crozier, E.; Jiang, D.; Frindt, R. Structures of Exfoliated Single Layers of $\mathrm{WS}_{2}, \mathrm{MoS}_{2}$, and $\mathrm{MoSe}_{2}$ in Aqueous Suspension. Phys. Rev. B 2002, 65, 1-9.

30. Prouzet, E.; Heising, J.; Kanatzidis, M. G. Structure of Restacked and Pillared $\mathrm{WS}_{2}$ : An X-ray Absorption Study. Chem. Mater. 2003, 15, 412-418.

31. Qin, X.; Yang, D.; Frindt, R.; Irwin, J. Scanning Tunneling Microscopy of Single-Layer $\mathrm{MoS}_{2}$ in Water and Butanol. Ultramicroscopy 1992, 42, 630-636.

32. Wypych, F.; Weber, T.; Prins, R. Scanning Tunneling Microscopic Investigation of $1 \mathrm{~T}-\mathrm{MoS}_{2}$. Chem. Mater. 1998, 10, 723-727.

33. Hansen, L. P.; Ramasse, Q. M.; Kisielowski, C.; Brorson, M.; Johnson, E.; Topsøe, H.; Helveg, S. Atomic-Scale Edge 
Structures on Industrial-Style $\mathrm{MoS}_{2}$ Nanocatalysts. Angew. Chem., Int. Ed. 2011, 50, 10153-10156.

34. Eda, G.; Fujita, T.; Yamaguchi, H.; Voiry, D.; Chen, M.; Chhowalla, M. Coherent Atomic and Electronic Heterostructures of Single-Layer $\mathrm{MoS}_{2}$. ACS Nano 2012, 6, 73117317.

35. Shi, Y.; Zhou, W.; Lu, A.-Y.; Fang, W.; Lee, Y.-H.; Hsu, A. L.; Kim, S. M.; Kim, K. K.; Yang, H. Y.; Li, L.-J.; et al. van der Waals Epitaxy of $\mathrm{MoS}_{2}$ Layers Using Graphene as Growth Templates. Nano Lett. 2012, 12, 2784-2791.

36. Komsa, H.-P.; Kotakoski, J.; Kurasch, S.; Lehtinen, O.; Kaiser, U.; Krasheninnikov, A. V. Two-Dimensional Transition Metal Dichalcogenides under Electron Irradiation: Defect Production and Doping. Phys. Rev. Lett. 2012, 109, 035503.

37. Algara-Siller, G.; Kurasch, S.; Sedighi, M.; Lehtinen, O.; Kaiser, U. The Pristine Atomic Structure of $\mathrm{MoS}_{2}$ Monolayer Protected from Electron Radiation Damage by Graphene. Appl. Phys. Lett. 2013, 103, 203107.

38. Zan, R.; Ramasse, Q. M.; Jalil, R.; Georgiou, T.; Bangert, U.; Novoselov, K. S. Control of Radiation Damage in $\mathrm{MoS}_{2}$ by Graphene Encapsulation. ACS Nano 2013, 7, 1016710174.

39. Treacy, M.; Howie, A. Z Contrast of Platinum and Palladium Catalysts. Philos. Mag. A 1978, 38, 569-585.

40. Brivio, J.; Alexander, D. T. L.; Kis, A. Ripples and Layers in Ultrathin $\mathrm{MoS}_{2}$ Membranes. Nano Lett. 2011, 11, 51485153.

41. Zhan, Y.; Liu, Z.; Najmaei, S.; Ajayan, P. M.; Lou, J. Large-Area Vapor-Phase Growth and Characterization of $\mathrm{MoS}_{2}$ Atomic Layers on a $\mathrm{SiO}_{2}$ Substrate. Small 2012, 8, 966-971.

42. Yang, D.; Frindt, R. F. Li-Intercalation and Exfoliation of $W_{2}$. J. Phys. Chem. Solids 1996, 57, 1113-1116.

43. Benavente, E.; Santa Ana, M. A.; González, G. Electrical Conductivity of $\mathrm{MoS}_{2}$ Based Organic-Inorganic Nanocomposites. Phys. Status Solidi 2004, 241, 2444-2447.

44. Tkatchenko, A.; Scheffler, M. Accurate Molecular van der Waals Interactions from Ground-State Electron Density and Free-Atom Reference Data. Phys. Rev. Lett. 2009, 102, 073005.

45. Zhu, Z. Y.; Cheng, Y. C.; Schwingenschlögl, U. Giant Spin-Orbit-Induced Spin Splitting in Two Dimensional Transition-Metal Dichalcogenide Semiconductors. Phys. Rev. B 2011, 84, 153402.

46. Mak, K. F.; He, K.; Shan, J.; Heinz, T. F. Control of Valley Polarization in Monolayer $\mathrm{MoS}_{2}$ by Optical Helicity. Nat. Nanotechnol. 2012, 7, 494-498.

47. Wu, S.; Ross, J. S.; Liu, G.-B.; Aivazian, G.; Jones, A.; Fei, Z.; Zhu, W.; Xiao, D.; Yao, W.; Cobden, D.; et al. Electrical Tuning of Valley Magnetic Moment through Symmetry Control in Bilayer MoS 2 . Nat. Phys. 2013, 9, 149-153.

48. Koch, C. T. QSTEM; http://elim.physik.uni-ulm.de/? page_id $=834$.

49. Blum, V.; Gehrke, R.; Hanke, F.; Havu, P.; Havu, V.; Ren, X.; Reuter, K.; Scheffler, M. Ab Initio Molecular Simulations with Numeric Atom-Centered Orbitals. Comput. Phys. Commun. 2009, 180, 2175-2196.

50. Perdew, J. P.; Burke, K.; Ernzerhof, M. Generalized Gradient Approximation Made Simple. Phys. Rev. Lett. 1996, 77, 3865-3868.

51. Marom, N.; Tkatchenko, A.; Scheffler, M.; Kronik, L. Describing Both Dispersion Interactions and Electronic Structure Using Density Functional Theory: The Case of MetalPhthalocyanine Dimers. J. Chem. Theory Comput. 2010, 6, 81-90.

52. Fernández-Seivane, L.; Oliveira, M. A.; Sanvito, S.; Ferrer, J. On-Site Approximation for Spin-Orbit Coupling in Linear Combination of Atomic Orbitals Density Functional Methods. J. Phys.: Condens. Matter 2006, 18, 7999-8013.

53. Soler, J. M.; Artacho, E.; Gale, J. D.; Garca, A.; Junquera, J.; Ordejón, P.; Sánchez-Portal, D. The SIESTA Method for $A b$ Initio Order-N Materials Simulation. J. Phys.: Condens. Matter 2002, 14, 2745-2779.

54. Ohto, T.; Rungger, I.; Yamashita, K.; Nakamura, H.; Sanvito, S. Ab-Initio Theory for Current-Induced Molecular Switching: Melamine on Cu(001). Phys. Rev. B 2013, 87, 205439. 